\title{
Multiple Assessment for Multiple Users in Virtual Reality Training Environments
}

\author{
Ronei M. Moraes ${ }^{1}$ and Liliane S. Machado ${ }^{2}$ \\ ${ }^{1}$ Department of Statistics \\ ${ }^{2}$ Department of Informatics \\ Universidade Federal da Paraíba \\ Cidade Universitária s/n - João Pessoa/PB - Brazil \\ ronei@de.ufpb.br, liliane@di.ufpb.br
}

\begin{abstract}
With recent computational advances, several interaction devices can be used by different users who share the same virtual world, allowing the simulation of realistic environments, such as surgical rooms. In order to deal with this feature, assessment systems must be generalized to evaluate, individually, all users of the simulation and to make the aspects of their interactions known. In this paper we propose a new assessment system for training based on virtual reality which can evaluate more than one user at a time. The methodology proposed uses data collected from user interaction and group interactions during training to create user profile and group profile. The main advantages of that approach are: both of reports can be used to increase group performance and the interactions among users, during training, can be monitored to correct and improve group tasks in procedure such as sequential, simultaneous or collaborative tasks.
\end{abstract}

Keywords: Multiple Assessment System, Training Based on Virtual Reality, Fuzzy Expert System, Statistical Measures, Statistical Models.

\section{Introduction}

The features found in training systems based on virtual reality can be the 3D environments composed of objects with topologies and behaviours similar to real objects, the interaction ways to deal with this world, the possibility of performing the training as much as necessary without risk or damage, among others [4]. Systems for different modalities in medicine have been developed as training in laparoscopy [23], bone marrow harvest [9], gynaecological exam [10] and bone surgery [17]. The goal of most of these systems is to provide a training environment similar to a real procedure environment by the use of devices and techniques which explore the human senses. All these systems can provide training for only one user at a time. However, some procedures need to be performed by more than one person.

Training systems based on VR for simultaneous use in complex training environments are being planned, as virtual surgery rooms for several purposes of training. The advances of cluster machines, improvements of video cards, processors and networks will provide training system for multiple users simultaneously in a few 
years at a low cost. Other important advance is the speed of input/output devices for virtual reality systems such as haptic devices. Nowadays, it is possible to connect more than one interaction device on a single computer.

Although the possibilities of training which can be simulated in VR systems, any kind of training has little value if the trainee does not have any feedback about his/her performance. Then, the existence of an on-line assessment tool attached to a simulation system based on VR is important to allow the learning improvement and the users assessment. This paper presents a brief overview of assessment in VR training systems for single users and proposes a method for assessment in collaborative training environments for simulators based on VR.

\section{Assessment in Virtual Reality Simulators}

The first methodologies for automatic assessment of training were proposed only a few years ago. This section presents a brief overview of assessment methodologies for training based on virtual reality. It can be noticed that several of them may also be potentially applied to other research areas. Basically, assessment methods can be divided in off-line and on-line. Off-line methods can be defined as methods not coupled to VR systems, whose assessment results are provided some time (which can be minutes, hours or days) after the end of the VR-based training. On the other hand, on-line assessment methods are coupled to the training system and collect user data to provide a result of his/her performance at the end of the simulation.

In medicine, some models for off-line or on-line assessment of training have been proposed. Some of them use Hidden Markov Models (HMM) [12, 19], fuzzy rulebased system [8], Fuzzy Gaussian Mixture Models [13], Evolving Fuzzy Neural Networks [14] and, recently, Fuzzy Bayes Rule [15]. An evaluation tool must continuously monitor all user interactions and compare his performance with predefined expert's classes of performance to recognize user's level of training. In spite of the methodologies proposed in literature, they are concerned with the assessment of only one user at a time. Up to the time being, methodologies which monitor multiple users in complex training environments based on virtual reality have not been found in literature.

\section{Multiple Assessment for Multiple Users in Virtual Reality Simulators}

Computational systems for multiple users have been developed since the 1990's, as RB2 [3], DIVE [5], MR Toolkit [20], some of them support haptic devices. Systems to provide interaction among multiple-user have been proposed too. Baier et al. [2] proposed a system for telepresence using haptic and visual interactions. Recently, systems for multi-user collaboration were developed [7, 16, 24]. The main differences of training systems based on virtual reality for multiple users are: increase of complexity of the virtual reality system - use of clusters of computers or a computer capable to generate realistic multiple views, support changes in virtual environments for multiple users and support assessment system; high speed peer-to-peer network 
for communication among computers without compromising the simulation. Eventually, more than one haptic device were installed in a computer and/or tracking systems for each user in training.

The most common problem in distributed systems based on network or Web, for multi-user interactions, is the latency [18]: users may have different views in the shared workspace which damages the users' performance involved in the simulation. For user assessment, the main problems related are the computational complexity and the accuracy, even when only one user is been trained. An on-line assessment system must have low complexity to does not compromise VR simulations performance, but it must have high accuracy to does not compromise the assessment.

Due to several specific necessities for multi-user training, a different approach for the assessment system is required. The requirements are: to monitor all users in training according to relevant variables to the training; in multi-user environment some tasks must be completed by specific users and according to a specific schedule; take measures of specific interactions among users during the time of simulation; take into account the length of assessment; to create a user profile and a group profile; to present low complexity not to compromise VR simulations performance, but present high accuracy level.

In the literature, some kinds of assessment have been proposed. Gande and Devarajan [6] have used an Instructor/Operator Station to monitor user movements and to increase or decrease the degree of difficulty in a simulation. Their Instructor Station is able to evaluate and monitor the resident's performance, based on the specifications mentioned, to generate training effectiveness reports. The GeRTiSS system [1] can provide the user an assessment report at the end of simulation. That report contains the total time of intervention, number of cuts and cauterisations, among other information. The surgeon can use these pieces of information to perform an assessment of his/her intervention.

\section{A Proposal for Multiple Assessment}

A tool for multiple assessment must be interconnected with all users and must receive from them synchronized information about all variables of interest. An assessment system works coupled to a virtual reality simulator, as showed in the Figure 1 [13]. As an on-line assessment system, which should be capable to monitor user interactions while he/she operates the simulation system, a multiple assessment must be capable to monitor, simultaneously, the interactions of each user. In order to reach that, it is necessary to collect information about position in the space, forces, torque, resistance, speeds, accelerations, temperatures, visualization and/or visualization angle, sounds, smells, etc. To collect some information as force, force feedback, angles and torques, it is necessary to use specific devices to provide them. This information will be collected for each user in training system, as well as for all groups, to be used to feed the assessment system. Additionally, synchronization in time and space is necessary for all users to measure interactions among them, to determine the ordering of tasks and to provide details of user's performance.

User's interactions with the system are monitored and the information are sent to the assessment system which analyses the data and emits, at the end of the training, an 
assessment report about the user's performance according pre-defined classes of performance. Therefore, a multiple assessment tool must be capable to monitor each user individually, as well as, all group. Then, at the end of simulation, another assessment report will be emitted about the group performance.

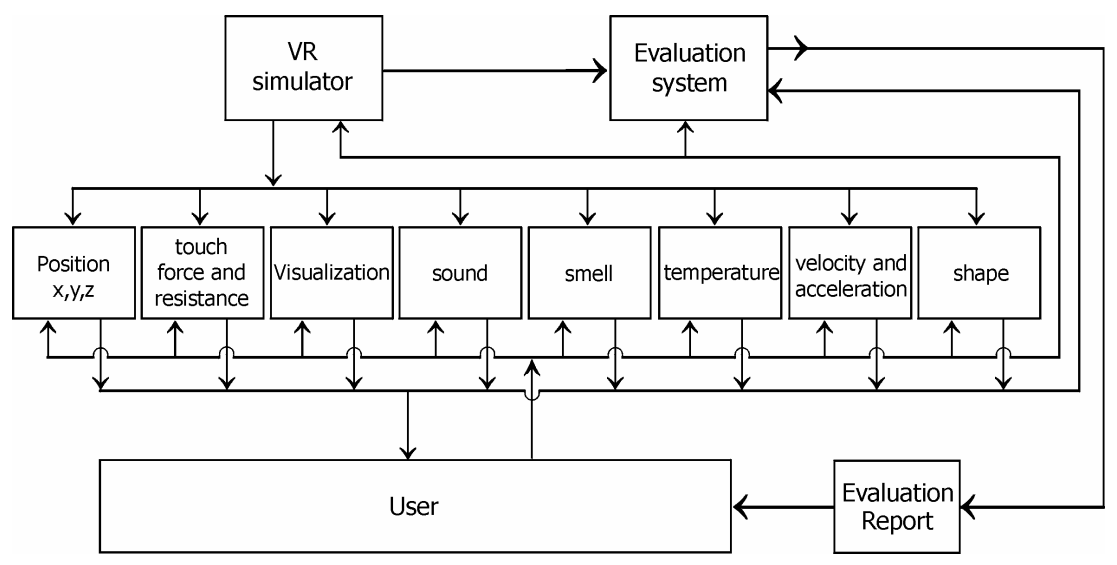

Fig. 1. Diagram of a VR simulator with an assessment system for a single user

As mentioned above, several methods were proposed to assess single user in training based on virtual reality. Most of them were based on classical classifiers. However, besides the fact that several of them could be used in multiple assessment tasks, there are calibration problems for multiple users. To minimize those problems, a multiple assessment system based on a fuzzy expert system [3, 11, 22] is proposed in this paper. This way, a set of fuzzy rules of an expert system time dependent defines each one of the possible performance classes. This set is designed, for single users and for group, from specialists knowledge. Additionally, interaction variables will be monitored according to their relevance to the training. Then, each application will have their own set of relevant variables which will be monitored [13]. The same happens with relevant variables which measure interactions among users in the group.

The methodology proposed for multiple assessment uses data collected from user interaction and group interactions during training to create user profile and group profile. That information is used to evaluate trainee and allows the improvement [21] of his performance in real tasks. That methodology makes a union of statistical tools and fuzzy rule based expert systems to construct an individual profile for trainee and for group. Statistical tools are programmed to make an automatic analysis of the database and construct statistical measures, tables, graphics and time dependent statistical models. From this information (statistical measures and parameters), the fuzzy expert system will create an individual user and group profiles and two kinds of report. These assessment reports present individual and group profiles and shows the performance of specific tasks with statistical measures, tables, graphics, models and some phrases in pseudo-natural language.

Figure 2 shows the new methodology presented. It can be observed that the Assessment System from Figure 1 has been changed and now it is called Individual 
Assessment System. To construct the Multiple Assessment System, the Group Assessment Tool, the Users and Group Profiles were also added. The $\mathrm{N}$ users perform their training using a VR Simulator and interacting with a Interactive System, which is responsible by management of the virtual environment. The Interactive System must provide visual and haptic simulations for all users according their point of view and their haptic devices. From these information, statistical measures and parameters are taking and they are used as input for a fuzzy expert system, which analyse that information to recognize user's and group levels of training.

At the end of training, the new Multiple Assessment System creates two kinds of report: individual assessment report, for each user, and group assessment report. The first report is about the individual user performance on the training and the second assessment report is about group performance and the interactions among users during training. That kind of interactions are monitored to correct and improve details in specific procedures, as sequential tasks, simultaneous tasks or collaborative tasks. These kind of tasks are common in surgical rooms and the group's performance in some tasks can be essentials for the life and the patient's recovery.

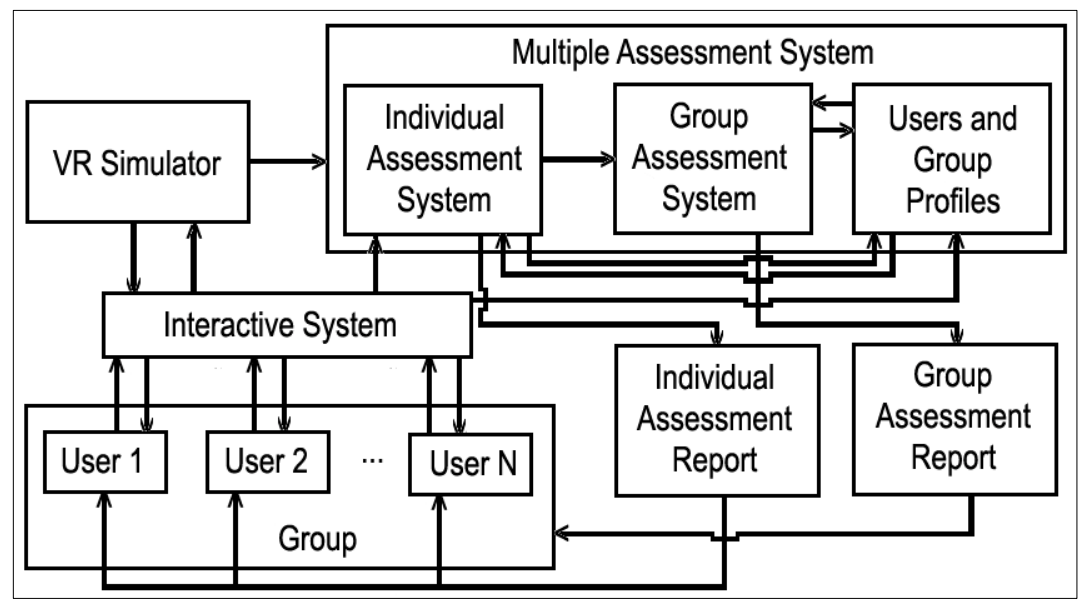

Fig. 2. Diagram of new Assessment System with approach of Continuous Assessment

This methodology for Multiple Assessment System can be used for several kind of training in medicine, as procedures in surgical rooms, training paramedics groups in emergency situations, etc. However, it is a generic methodology and can be used in training systems for other areas, as aeronautical simulators, maintenance group, managing of nuclear, thermoelectric, hydroelectric power plant, etc.

However, this Multiple Assessment System cannot be classified as on-line or offline as in single user assessment systems. As mentioned before, an on-line assessment system must generate reports immediately after of the end of training session. Sometimes, the Multiple Assessment System will be capable to do that, but in another cases it will be not. The main reasons for that involve: computers configuration, possible delay in networks, number of statistical time dependent models necessary to measure some variables, complexity of simulation and number of interactions. 


\section{Conclusions}

This paper presents a review for assessment systems for training based on virtual reality for single user. It was presented also the future of training based on virtual reality for multiple users with interactions among them. For this, are necessary new methodologies to allow the assessment of training for each user as well as for the group. In order to do that, was introduced a new methodology for multiple assessment for multiple users in training. This methodology is based on time-dependent fuzzy based rules expert system which uses input variables from training, statistical measures and time dependent statistical models in order to create measures of assessment for trainees and group performance.

The Multiple Assessment System can create two kinds of report: an assessment report for individual user, about his/her performance on the training, and an assessment report about group performance and interactions among users during the training. The Multiple Assessment System can be used in training situations when a group of trainees performs a task together, as in surgical rooms.

The methodology is generic and can be used in training systems for other areas, as aeronautical simulators, maintenance group, managing of nuclear, thermoelectric, hydroelectric power plant, etc. The main advantages of that approach are: a) individuals and group reports can be used to increase group performance and b) the interactions among users during training can be monitored to correct and improve specific details in procedure such as sequential tasks, simultaneous tasks or collaborative tasks.

\section{References}

1. Alcañiz, M., et al.: GeRTiSS: Generic Real Time Surgery Simulation. Studies in Health Technology and Informatics 94, 16-18 (2003)

2. Baier, H.: Distributed PC-based haptic, visual and acoustic telepresence system experiments in virtual and remote environments. In: Proc. of IEEE VR, USA, pp. 118-125 (1999)

3. Blanchard, C., et al.: Reality built for two: a virtual reality tool. ACM CG 24(2), 35-36 (1992)

4. Burdea, G., Coiffet, P.: Virtual Reality Technology, 2nd edn. Wiley, Chichester (2003)

5. Carlsson, C., Hagsand, O.: DIVE: a platform for multi-user virtual environments. Computers \& Graphics 17(6), 663-669 (1993)

6. Gande, A., Devarajan, V.: Instructor station for virtual laparoscopic surgery: requirements and design. In: Proc. of Computer Graphics and Imaging, USA, pp. 85-90 (2003)

7. Low, K-L., et al.: Combining head-mounted and projector-based displays for surgical training. In: Proc. of the IEEE VR, USA, pp. 110-117 (2003)

8. Machado, L.S., et al.: Fuzzy Rule-Based Evaluation for a Haptic and Stereo Simulator for Bone Marrow Harvest for Transplant. In: 5th PUG Workshop Proc. USA (2000)

9. Machado, L.S., et al.: A Virtual Reality Simulator for Bone Marrow Harvest for Pediatric Transplant. Studies in Health Technology and Informatics 81, 293-297 (2001)

10. Machado, L.S., Moraes, R.M.: Online Training Evaluation in Virtual Reality Simulators Using Evolving Fuzzy Neural Networks. In: Proc. 6th FLINS Conference. Belgium, pp. 314-317 (2004) 
11. Machado, L.S., Valdek, M.C.O., Moraes, R.M.: Assessement of Gynecological Procedures in a Simulator Based on Virtual Reality. In: Proc. of the 7th FLINS Conference. Italy, pp. 799-804 (2006)

12. Moraes, R.M., Machado, L.S.: Using Fuzzy Hidden Markov Models for Online Training Evaluation and Classification in Virtual Reality Simulators. International Journal of General Systems 33(2-3), 281-288 (2004)

13. Moraes, R.M., Machado, L.S.: Fuzzy Gaussian Mixture Models for On-line Training Evaluation in Virtual Reality Simulators. In: FIP 2003, China, vol. 2, pp. 733-740 (2003)

14. Moraes, R.M., Machado, L.S.: Evaluation System Based on EFuNN for On-line Training Evaluation in Virtual Reality. In: Sanfeliu, A., Cortés, M.L. (eds.) CIARP 2005. LNCS, vol. 3773, pp. 778-785. Springer, Heidelberg (2005)

15. Moraes, R.M., Machado, L.S.: On-line Training Evaluation in Virtual Reality Simulators using Fuzzy Bayes Rule. In: Proc. 7th FLINS Conference, Italy, pp. 791-798 (2006)

16. Morris, et al.: A Collaborative Virtual Environment for the Simulation of Temporal Bone Surgery. In: Proc. MICCAI, France, pp. 319-327 (2004)

17. Morris, et al.: Visuohaptic simulation of bone surgery for training and evaluation. IEEE Computer Graphics and Applications 26(6), 48-57 (2006)

18. Park, K-S., Kenyon, R.V.: Effects of network characteristics on human performance in a collaborative virtual environment. In: Proc. of IEEE Virtual Reality, USA, pp. 104-111 (1999)

19. Rosen, J., Solazzo, M., Hannaford, B., Sinanan, M.: Objective Laparoscopic Skills Assessments of Surgical Residents Using Hidden Markov Models Based on Haptic Information and Tool/Tissue Interactions. Studies in Health Technology and Informatics 8, 417-423 (2001)

20. Shaw, C., Green, M.M.: Toolkit peers package and experiment. Proc. IEEE VRAIS, USA, pp. 463-469 (1993)

21. Sternberg, R.J., Grigorenko, E.: Dynamic Testing: The Nature and Measurement of Learning Potential. Cambridge University Press, Cambridge (2001)

22. Terano, T., Asai, K., Sugeno, M.: Fuzzy systems theory and it's applications. Academic Press Inc. San Diego (1987)

23. Voss, G., et al.: Intelligent Training System for Laparoscopy and Hysteroscopy. Studies in Health Technology and Informatics (70), 359-364 (2000)

24. Yoshida, S., Noma, H., Hosaka, K., Proactive Desk, I.I.: Development of a New Multiobject Haptic Display Using a Linear Induction Motor. In: Proc. IEEE VR Conference, USA, pp. 269-272 (2006) 